\title{
Lipoinyección Labial con Grasa Autóloga con fines Estéticos.
}

\author{
Miguel Andrés Montalvo Clavijo ${ }^{1 *}$, Dayamí Zaldívar Castillo², Clara Elena Peñalver Rodríguez ${ }^{3}$.
}

\begin{abstract}
1Doctor en medicina. Especialista en Cirugía Plástica y Caumatología. Hospital Clínico Quirúrgico "General. Calixto García". La Habana. Cuba. ${ }^{2}$ Doctora en medicina. Especialista en primer grado Cirugía Plástica Caumatología. Especialista en primer grado en Medicina General Integral. Master en Ciencias. Profesor asistente. Hospital Clínico Quirúrgico "General. Calixto García”. La Habana. Cuba ${ }^{3}$ Doctora en medicina. Profesora Titular. Hospital Clínico Quirúrgico "General. Calixto García”. La Habana. Cuba
\end{abstract}

\section{RESUMEN}

Introducción. La Lipoinyección de grasa autóloga con fines estéticos es una de las técnicas más jóvenes que permiten resolver problemas relacionados con valores concernientes a las elecciones personales que apuntan hacia la belleza, es un procedimiento quirúrgico sencillo y con resultados rápidos. Es una alternativa segura, real, sencilla y accesible que abarata costos, sobre todo con el uso de equipos y tecnología.

Objetivos. Caracterizar los resultados estéticos de la Lipoinyección Labial con Grasa Autóloga en pacientes que asistieron a la consulta externa de Cirugía Plástica del Hospital Universitario "General Calixto García".

Material y Método. Se realizó un estudio prospectivo, descriptivo, de corte transversal, en el período comprendido entre octubre de 2018 a septiembre del 2019. Se seleccionaron aquellos que cumplieron los criterios de inclusión y aceptaran a participar en el estudio voluntariamente. La muestra quedó constituida por 23 pacientes. La información obtenida se procesó mediante bases de datos confeccionadas en Excel, lo que permitió la realización de la estadística descriptiva simple para las variables individuales como media, desviación estándar y razón. La disposición a colaborar en el estudio se recogió en el modelo de Consentimiento Informado elaborado para esta investigación.

Resultados. predominio del sexo femenino en el $60.87 \%$ de los operados. El grupo de edad entre 51 a 61 años fue el de mayor número de pacientes. Las complicaciones inmediatas más frecuentes en los labios fueron: la inflamación y los hematomas; las tardías fueron nodulaciones y endurecimiento. El $82.60 \%$ de los pacientes se mostró satisfecho con los resultados.

Conclusiones. La Lipoinyección labial con Grasa Autóloga se caracterizó por ser un método capaz de satisfacer el resultado estético. Las variables edad y sexo evidencian que las mujeres se realizaron más Cirugía Estética Labial que los hombres. Las variables clínicas labios finos y volumen evidencian que el aumento de la edad es una condición importante que se asocia a la demanda de esta técnica quirúrgica y que el ser hombre o mujer no establece una diferencia para la solitud de este tipo de técnica quirúrgica. Se presentaron complicaciones inmediatas y tardías. Los niveles de satisfacción expresados por los pacientes fueron altos.

Palabras clave: Cirugía estética, Lipoinyección, grasa autóloga, LabioHipotrófico.

\section{ABSTRACT}

\section{Autologous fat Lipoinjection for Aesthetic Purposes.}

Introduction. Autologous fat lipoinjection for aesthetic purposes is one of the youngest techniques that allow solving problems related to values concerning personal choices that point towards beauty, it is a simple surgical procedure with quick results. It is a safe, real, simple and accessible alternative that lowers costs, especially with the use of equipment and technology,

Objectives. To characterize the aesthetic results of Lip Lipoinjection with Autologous Fat in patients who attended the Plastic Surgery outpatient clinic at the "General Calixto García" University Hospital.

Material and Method. A prospective, descriptive, cross-sectional study was conducted in the period from October 2018 to September 2019. Those who met the inclusion criteria and voluntarily agreed to participate in the study were selected. The sample consisted of 23 patients. The information obtained was processed through databases prepared in Excel, which allowed the performance of simple descriptive statistics for individual variables such as mean, standard deviation and ratio. The willingness to collaborate in the study was included in the Informed Consent model prepared for this research.

Results. female predominance in $60.87 \%$ of those operated on. The age group between 51 and 61 years was the one with the largest number of patients. The most frequent immediate complications on the lips were: inflammation and bruising; the later ones were nodulations and hardening. $82.60 \%$ of the patients were satisfied with the results.

Conclusions. Lip Lipoinjection with Autologous Fat was characterized as a method capable of satisfying the aesthetic result. The age and sex variables show that women underwent more cosmetic lip surgery than men. The clinical variables thin lips and volume show that increasing age is't a condition that is associated with the demand for this technique and that the Being male or female does not make a difference to the solitude of this type of surgical technique. There were immediate and late complications. The satisfaction levels expressed by the patients were high.

Keywords: Cosmetic surgery, Lipoinjection, autologous fat, Hypotrophic Lip.

*Autor de correspondencia: Miguel Andrés Montalvo Clavijo andresmick@hotmail.com

Como citar: Montalvo Clavijo MA, Zaldivar Castillo D, Peñalver Rodriguez CE. Lipoinyección labial con grasa autóloga con fines estéticos. Revista Cuarzo 2020;26(2):18-26.

Recibido: 22 de octubre de 2020 Aceptado: 15 de noviembre de 2020 Publicado: 30 de diciembre de 2020

DOI: https://doi.org/10.26752/cuarzo.v26.n2.505

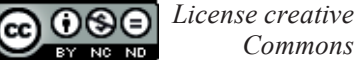

${ }^{1}$ Esta investigación constituyó el trabajo de tesis para optar por el grado de especialista en primer grado Cirugía Plástica y Caumatología. 


\section{INTRODUCCIÓN}

E En términos generales, se define a la Cirugía Plástica, EEstética, Reparadora o Reconstructiva, como la especialidad quirúrgica encargada de restablecer la integridad anatómica o funcional del cuerpo humano, alterado por defectos físicos, congénitos o adquiridos(1).

Largo ha sido el recorrido histórico de la Cirugía Plástica (CP) especializada, esta comienza a aparecer alrededor del año 1950 a.c. con los babilonios, existen evidencias de que, en la India, Sushruta (600 a.c.) ya se describen y realizan operaciones para la reconstrucción nasal y de los lóbulos auriculares, utilizando incluso colgajos cuyos principios se conservan en la técnica indiana, utilizada actualmente(2). Ya en un acercamiento en el siglo $\mathrm{XX}$, hay un punto crucial en el desarrollo de la CP, que es la Primera Guerra Mundial, por la gran cantidad de pacientes que había que reconstruir y rehabilitar, a los cuales la simple amputación no era una solución para sus problemas. Hacia 1930 se inició a nivel mundial la organización integral de servicios de Cirugía Plástica en los hospitales, que incluían en sus actividades el manejo de heridas, quemaduras, la realización de injertos, colgajos, cirugías de la mano, cirugías maxilofacial, reconstructiva y estética(2).

Ya más contemporáneo, el siglo XXI, se han marcado y redefinido pautas estéticas, ya no basadas en correcciones solo congénitas o adquiridas, la estética de hoy pasa y apunta también a valores estéticos desde elecciones personales sin que haya lesión previa

La Sociedad Internacional de Cirugía Plástica Estética, conocida por sus siglas en inglés (ISAPS), evidenció en un reciente estudio que: "la Cirugía Estética continúa aumentando en todo el mundo, el total de procedimientos quirúrgicos y no quirúrgicos aumentó en 5,4\% en 2018 respecto al año 2015”(3).

En el mundo contemporáneo la alta demanda de cirugías estéticas ha generado la inclusión de muchos abordajes quirúrgicos y no quirúrgicos, una de las técnicas quirúrgicas más jóvenes es la Lipoinyección con Grasa Autóloga sobre la cual versará esta investigación. Intentar satisfacer demandas contextuales desde lo académico reviste una gran importancia y actualidad, de ahí que realizar un trabajo de investigación sobre uso de la Lipoinyección con Grasa Autóloga con fines estéticos en Labios Hipotróficos sea una alternativa eficaz además de una necesidad investigativa que permitirá poder sistematizar un conocimiento que bibliográficamente es escaso de modo que sirva a futuros especialistas desde una visión teórico y práctico. Además de todas las ventajas que implica el uso de esta técnica, entre las cuales están(4-6):

- Ser una alternativa segura, real, sencilla y accesible que abarata costos, sobre todo con el uso de equipos $y$ tecnología

- La grasa autóloga se puede definir como un excelente elemento de relleno y el menos perjudicial para el cuerpo, ya que emplea el tejido adiposo del propio paciente.

- Esta técnica no sólo aporta volumen de forma duradera y del todo compatible al ser nuestra propia grasa, sino que, además, mejora la calidad de la piel dándole más tono, firmeza y luminosidad.

- Los resultados son estables y duraderos si el tratamiento es bien hecho, de rápida recuperación $\mathrm{y}$ marcas imperceptibles.

Por tanto, el propósito general de la siguiente investigación es caracterizar los resultados estéticos de la Lipoinyección Labial con Grasa Autóloga en pacientes que asistieron a la consulta externa de Cirugía Plástica del Clínico Quirúrgico "General Calixto García” en La Habana Cuba.

\section{MATERIAL Y MÉTODOS}

Se realizó un estudio prospectivo, descriptivo y de corte transversal a los pacientes que asistieron a la consulta externa de Cirugía Plástica del Hospital Universitario Calixto García en el período comprendido entre octubre del 2018 a septiembre del 2019.

De todos los pacientes que asistieron a la consulta de Cirugía Plástica de dicho hospital en el período antes mencionado y que solicitaron un aumento labial, se seleccionaron aquellos que cumplieron los criterios de inclusión y aceptaran a participar en el estudio voluntariamente. La muestra quedó constituida por 23 pacientes que asistieron a la consulta de Cirugía Plástica solicitando una cirugía para aumento labial.

\section{Criterios de Inclusión:}

- Pacientes que soliciten la realización de la Queiloplastia con Labio Hipotrófico.

- Pacientes de ambos sexos.

- Pacientes comprendidos entre las edades de 18 años hasta 61 años.

- Pacientes que no posean hábito de fumar, ni sean ex fumadores recientes.

- Paciente que tenga todos los complementarios en la consulta pre quirúrgico dentro de los valores normales.

- Brindar su consentimiento a participar en la investigación.

\section{Criterios de Exclusión:}

- Presentar algún tipo de discapacidad genética o entidad psiquiátrica que impida participar en la investigación.

- Pacientes con enfermedades crónicas descompensadas.

- Pacientes con antecedentes de trastornos congénitos o cicatriciales deformantes.

- Patologías infecciosas bucales y/o trastornos dermatológicos en zonas donantes o receptoras.

- Hipersensibilidad o alergia a la lidocaína o epinefrina conocida.

- Expresar su deseo de abandonar el estudio en cualquier fase del mismo.

- Brindar su consentimiento a participar en la investigación 


\section{Variables Sociodemográficas}

Edad del paciente, expresada en años cumplidos, se agruparon en rangos de: 18-28 años, 29-39 años, 40-50 años y 51-61 años.

Sexo: Según sexo biológico: Masculino y Femenino.

Clínicas: Complicaciones zona donante y receptora en términos de aparición de algún signo o síntoma de complicación en el posoperatorio: Protuberancias, Endurecimiento, Cicatriz, Dehiscencia, Infección local, Asimetrías, Esteatonecrosis, Seroma, Eritemas, Hematomas, Hipertermia

Edema, Nódulos, Hipersensibilidad.

Tiempo de Recuperación: complicaciones post operatorias inmediatas.

Defecto labial; Labio Fino. Labio fino: Hombres y mujeres que estuviesen por debajo de los siguientes parámetros establecidos como normales:

Labio: longitud vertical lateral.

- Hombres 11-21 mm

- Mujeres 10-20 mm.

Media o central.

- Hombres 18-26 mm

- Mujeres 16-24 mm. No obstante

Volumen: Los labios tienen pérdida de volumen al pasar de los años por la pérdida de la producción de colágeno y elastina fundamentalmente, de ahí que se tomó como norma el criterio subjetivo, la designación personal de aumentar el grosor desde dicha elección.

Nivel de Satisfacción expresada en:

\section{Variables Socio psicológicas}

- Satisfecho: paciente que se muestra y expresa satisfacción por el resultado logrado en términos de expectativas y satisfacción de sus necesidades estéticas.

- Insatisfecho: paciente que no está conforme estéticamente con el resultado final y Medianamente satisfecho

\section{Método de recolección de información.}

1- Se confeccionó por el autor una planilla que permitió recolectar los datos acerca del procedimiento de Lipoinyección Labial con Grasa Autóloga permitiendo enunciar los aspectos fundamentales de este proceder.

2- Se creó por el autor una encuesta que permitió identificar el nivel de satisfacción de estos pacientes con los resultados a este tipo Cirugía Plástica con la técnica de Lipoinyección Labial con Grasa Autóloga, la misma abarca aspectos como: calidad y satisfacción con la información médica recibida, forma, volumen, asimetría, consistencia y valoración global con el resultado. Para la confección de la misma se tuvo en cuenta los aspectos a medir referidos por otros autores en este tipo de cirugía(7-9).

\section{Procedimiento general.}

Los pacientes acudieron a una primera consulta solicitando una modificación estética de sus labios faciales. Y se siguieron los siguientes pasos en esta:

Examen físico facial que contempló la medición de los labios con los parámetros ya descritos utilizándose un Pie de Rey. Respecto al volumen la medida utilizada fue la del criterio personal, es decir se respetó la auto percepción del volumen de sus labios, razón por la que se toma como un defecto labial desde la subjetividad del paciente.

$\checkmark$ Entrevista médica, para conocer si son o no tributarios del proceder a emplear, debiendo cumplir con los criterios de inclusión. Se les explica en qué consiste el proceso a que pretenden someterse, más las pruebas complementarias (hemograma completo, glicemia, creatinina, VIH, serología, coagulograma y electrocardiograma a mayores de 40 años) y se les entrega el consentimiento informado en el cual esta descrito todo el proceder médico a emplear, las pruebas y los riesgos que pudieran ocurrir e incluye el permiso para hacer unas fotografias iniciales que después servirán como muestra del resultado.

Se les pide una valoración por escrito de un odontólogo y un dermatólogo que haga constar que no tienen ningún impedimento físico para someterse al procedimiento de Lipoinyección labial. Si el paciente está de acuerdo, se le cita para una segunda consulta.

En la segunda consulta se evalúan los resultados de los complementarios, dermatología y odontología, de encontrarse dentro de los parámetros normales se le informa y se realizan las fotografías iniciales. En esta misma consulta se anotan en su historia clínica ambulatoria y además se adjunta una planilla creada al uso de esta investigación que reúne los siguientes parámetros:

Tipo de Lipoinyección: Es de pequeño volumen $(<100$ $\mathrm{mL})$.

$\square$ Tipo de anestesia: Mediante técnica de asepsia y antisepsia, se utilizó anestesia local en todos los pacientes con una solución consistente a la descrita por Klein: se infiltró $(50 \mathrm{~mL}$ de solución salina a $0.9 \%, 5 \mathrm{~mL}$ de bicarbonato de sodio y $10 \mathrm{~mL}$ de lidocaína con epinefrina a $2 \%$ ), mediante anestesia tumescente, en la zona de abdomen inferior y papada. Para lograr el bloqueo de la región de los labios superiores e inferiores, se infiltran de 3 a $5 \mathrm{~mL}$ de anestésico (Lidocaína $2 \%$ ).

Zona de la cual se extrae la grasa autóloga: Se extrajo del abdomen inferior y papada. Se realizó la marcación preoperatoria con los pacientes, empleando diferentes colores: uno para la zona a Lipoaspirar, y otro en las zonas a aplicar la lipotransferencia (Lipoinyección).

$\square$ Procesamiento y obtención del tejido graso: Se utilizó el método de decantación por gravedad: se intenta producir el menor trauma posible al adipocito por este motivo se usa la aspiración manual mediante jeringas de una capacidad no mayor a los $10 \mathrm{ml}$ conectadas a cánulas de un diámetro de $15 \mathrm{~cm}$ de largo por $3 \mathrm{~mm}$ de ancho con 3 orificios a un costado sin exceder el efecto de la presión negativa sobre el adipocito, posibilitando la extracción de 
parcelas de grasa intactas en un estado óptimo para su revascularización en el lecho receptor.

Lipoinyección e infiltración de grasa autóloga en el labio: El tejido graso se inyectó en el área del labio a nivel del tejido celular subcutáneo mediante una cánula roma con un solo orificio de salida de infiltración de $5 \mathrm{~cm}$ de largo por $1.5 \mathrm{~mm}$ de ancho. La cantidad a inyectar se individualizó para cada paciente y se registró el volumen inyectado.

- Postoperatorio: Posterior a la culminación de la cirugía se realizó la evolución médica completa y se mantendrá en observación por 1 hora en posición decúbito supino para no alterar el resultado de la Lipoinyección. Se otorgó el alta hospitalaria con las indicaciones correspondientes, analgésicos, compresas frías y antibiótico profiláctico.

Seguimiento: Al mes con examen físico y control fotográfico, a los tres meses (se aplica la encuesta de satisfacción) más control fotográfico y a los 6 meses de ser necesario, por insatisfacción del resultado.

\section{Método de recolección de información.}

Esta investigación se realizó teniendo en cuenta los principios éticos planteados en el Código de Núremberg y la Declaración de Helsinki para las investigaciones médicas, en la cual como uno de los aspectos más importantes planteaba(10): “En la investigación médica, es deber del médico proteger la vida, la salud, la intimidad y la dignidad del ser humano... beneficios calculados, riesgos previsibles e incomodidades derivadas del experimento. La persona debe ser informada del derecho de participar o no en la investigación y de retirar su consentimiento en cualquier momento, sin exponerse a represalias. Después de asegurarse de que el individuo ha comprendido la información, el médico debe obtener entonces, preferiblemente por escrito, el consentimiento informado $y$ voluntario de la personall".

La disposición a colaborar en el estudio se recogió en el modelo de Consentimiento Informado elaborado para esta investigación. Los demás aspectos éticos a tener en cuenta en esta investigación se basan en la no divulgación de nombres, hechos, situaciones que acontezcan durante el procedimiento o posteriores a este, no se hará alusión a casos particulares. Las Fotografías que se utilizarán en los anexos, se procesarán de manera que no se mostrará la identidad del paciente.

Para la realización de este trabajo, antes del inicio de la investigación, fue valorado por el Comité de Ética de la Facultad de Ciencias Médicas de La Habana, Hospital Universitario "Gral. Calixto García el cual autorizó la realización de la misma.

\section{RESULTADOS}

La tabla No. 1 muestra el universo de pacientes que solicitaron tratamiento de Queiloplastia en la consulta externa de Cirugía Plástica del Hospital Universitario "Calixto García" en el período de octubre 2018 a septiembre 2019. Se atendieron 37 pacientes, de los cuales 26 son del sexo femenino lo que representa el $70.27 \%$ del total y $11(29.72 \%)$ son masculinos.
Al realizarse el cálculo de la razón entre mujeres y hombres se obtuvo que esta población tenía 2.36 mujeres por cada hombre que solicitó atención médica para Cirugía Plástica.

Tabla 1: Casos atendidos en la consulta externa de Cirugía Plástica del Hospital Universitario "Calixto García" en el período comprendido entre octubre del 2018 a septiembre del 2019.

\begin{tabular}{cccccc}
\hline \hline \multicolumn{5}{c}{ SEXO } \\
\hline & Masculino & \multicolumn{2}{c}{ Femenino } & \multicolumn{2}{c}{ Total } \\
\hline \hline $\mathbf{N}$ & $\%$ & $\mathrm{~N}$ & $\%$ & $\mathrm{~N}$ & $\%$ \\
\hline $\mathbf{1 1}$ & 29.72 & 26 & 70.27 & 37 & 100 \\
\hline \hline \multicolumn{5}{c}{ Elaboración propia. ${ }^{*}$ Cálculo de la razón mujeres-hombres }
\end{tabular}

La tabla No. 2 permite ver la descripción de la muestra distribuida por grupos de edad y sexo, se observa que de los 23 pacientes que asistieron a la consulta de Cirugía Plástica del Hospital Universitario "Calixto García” y cumplieron con los criterios para ser incluidos en la muestra de esta investigación, el mayor número de pacientes se encuentra en el grupo de 51 a 61 años de edad y representan el $39.13 \%$ de la muestra de los cuales $5(21.73 \%)$ son masculinos $4(17.39 \%)$ son femeninos, en segundo lugar está el grupo de 29 a 39 años de edad con igual número de pacientes masculinos y femeninos 3(13.04\%) respectivamente. El tercer lugar de la muestra lo ocupa el grupo de edad entre 18 a 28 años, solo representado por el sexo femenino con $5(21.74 \%)$ pacientes. El último lugar por grupos de edades está el de 40 a 50 años con 1(4.34\%) paciente masculino y $2(8.70 \%)$ femeninas.

Tabla 2: Distribución por edad y sexo de los pacientes atendidos por Cirugía Plástica para aumento labial.

\begin{tabular}{|c|c|c|c|c|c|c|}
\hline \multirow{3}{*}{ Edad } & \multicolumn{4}{|c|}{ SEXO } & \multirow{2}{*}{\multicolumn{2}{|c|}{ Total }} \\
\hline & \multicolumn{2}{|c|}{ Masculino } & \multicolumn{2}{|c|}{ Femenino } & & \\
\hline & $\mathrm{N}$ & $\%$ & $\mathrm{~N}$ & $\%$ & $\mathrm{~N}$ & $\%$ \\
\hline $18-28$ & 0 & 0.00 & 5 & 21.74 & 5 & 21.74 \\
\hline $29-39$ & 3 & 13.04 & 3 & 13.04 & 6 & 26.09 \\
\hline $40-50$ & 1 & 4.34 & 2 & 8.70 & 3 & 13.04 \\
\hline $51-61$ & 5 & 21.73 & 4 & 17.39 & 9 & 39.13 \\
\hline Total & 9* & 39.13 & $14^{*}$ & 60.87 & 23 & 100 \\
\hline
\end{tabular}

El total de la muestra apunta a que el $60.87 \%$ son femeninos y el $39.13 \%$ masculinos, la edad promedio de los pacientes estudiados fue de 42.17 años y el cálculo de la razón entre mujeres y hombres fue de 1.56 mujeres por cada hombre, lo que muestra que existe una diferencia discreta que apunta hacia haber más pacientes del sexo femenino que del masculino en "casi" el doble, la mediana fue de 40 años lo que nos dice que el $50 \%$ de los sujetos está por encima de los 40 años de edad y el otro $50 \%$ por debajo.

La tabla No.3 describe los defectos labiales encontrados en la muestra estudiada, sean estos asociados a la pérdida de volumen o a tener labios finos o a la combinación de ambos. 


\section{2 | Pá g i n a}

Con defecto labial por labios finos se encuentra en los grupos de edades comprendidas entre los 18 a 28 y 29 a 39 años con $5(21.73 \%)$ pacientes respectivamente, de ellos 7 son femeninos y 3 masculinos En segundo lugar se encuentra el grupo de 40 a 50 años de edad con 2 paciente que representan el $8.69 \%$ de la muestra, uno femenino y otro masculino. El grupo de 51 a 61 años de edad no presentó este defecto labial.

Tabla 3: Defecto Labial.

\begin{tabular}{ccccccc}
\hline \hline \multirow{2}{*}{$\begin{array}{c}\text { Rango de } \\
\text { edad en } \\
\text { Años }\end{array}$} & \multicolumn{3}{c}{ Labio Finos } & $\%$ & \multicolumn{4}{c}{$\begin{array}{c}\text { Vérdida de } \\
\text { Volumen }\end{array}$} & \multirow{2}{*}{$\%$} \\
\cline { 2 - 5 } & $\mathrm{M}$ & $\mathrm{F}$ & & $\mathrm{M}$ & $\mathrm{F}$ & \\
\hline \hline 18 a 28 & 0 & 5 & 22 & 0 & 0 & 0 \\
29 a 39 & 3 & 2 & 22 & 0 & 1 & 4.34 \\
40 a 50 & 1 & 1 & 9 & 0 & 1 & 4.34 \\
51 a 61 & 0 & 0 & 0 & 5 & 4 & 39.13 \\
\hline \hline TOTAL & $\mathbf{4}$ & $\mathbf{7}$ & $\mathbf{4 7 , 8 3}$ & $\mathbf{5}$ & $\mathbf{6}$ & $\mathbf{4 7 , 8 3}$ \\
\hline \hline
\end{tabular}

La pérdida de volumen estuvo presente en los grupos de 29 a 39 años y en el de 40 a 50 años con 1(4.34\%) paciente respectivamente del sexo femenino. El grupo de edad comprendida entre 51 a 61 años fue el de mayor número de pacientes con pérdida de volumen expresada como un defecto labial y estuvo representado por 9 pacientes lo que equivale al $39.13 \%$ del total de la muestra, siendo 5 de masculinos y 4 femeninos.

La Tabla No.4 muestra que de los 23 pacientes operados 3 presentaron síntomas inmediatos, 1 presentó inflamación aguda en ambos labios, uno presentó este mismo síntoma en el labio superior y otro paciente presentó un hematoma en el labio inferior. Representando estos tres pacientes el $13.04 \%$ del total de la muestra.

Tabla 4: Complicaciones inmediatas evaluadas a las 24 horas del post operatorio.

\begin{tabular}{ccccc}
\hline \hline Complicaciones & $\begin{array}{c}\text { Labio } \\
\text { Superior }\end{array}$ & $\%$ & $\begin{array}{c}\text { Labio } \\
\text { Inferior }\end{array}$ & $\%$ \\
\hline \hline $\begin{array}{c}\text { Reacciones inflamatorias } \\
\text { agudas *** }\end{array}$ & 2 & 8.69 & 1 & 4.34 \\
Hematomas** & 0 & 0 & 1 & 4.34 \\
\hline \hline Total, $\mathbf{N}=\mathbf{2 3}$ & $\mathbf{2}$ & $\mathbf{8 . 6 9}$ & $\mathbf{2}$ & $\mathbf{8 . 6 9}$
\end{tabular}

Elaboración propia $* * *$ Solo un mismo paciente presentó una reacción inflamatoria aguda para ambo labios, el otro paciente presentó este síntoma solo en el labio superior. ** El hematoma, lo presentó un paciente como un único síntoma.

La tabla No. 5 muestra las complicaciones tardías evaluadas a los 30 días de haber sido operados, 2(8.69\%) pacientes presentaron Hipersensibilidad en el labio inferior y $1(4.34 \%)$ presentó endurecimiento en el labio superior y otro en el labio inferior y uno presentó nodulaciones en el labio superior. Siendo 5 los pacientes que presentaron complicaciones tardías, lo que representan el $21.73 \%$ del total de los pacientes operados.
Revista Cuarzo (2020) volumen $26-2$ Montalvo Clavijo MA et al.

Tabla No.6. Evaluación del Nivel de satisfacción presentado por los pacientes operados por Lipoinyección con Grasa Autóloga.

Tabla 5: Complicaciones tardías evaluadas a los 30 días del postoperatorio.

\begin{tabular}{|c|c|c|c|c|c|c|}
\hline "Complicaciones & $\begin{array}{c}\text { Labio } \\
\text { Superior }\end{array}$ & $\%$ & $\begin{array}{c}\text { Labio } \\
\text { Inferior }\end{array}$ & $\overline{\%}$ & total & $\overline{\%}$ \\
\hline Hipersensibilidad & 0 & 0 & 2 & 8.69 & 2 & 8.69 \\
\hline Endurecimiento & 1 & 4.34 & 1 & 4.34 & 2 & 8.69 \\
\hline Nodulaciones & 1 & 4.34 & 0 & 0 & 1 & 4.34 \\
\hline Total & 2 & 8.69 & 3 & 13.03 & 5 & 21.73 \\
\hline
\end{tabular}

Tabla 6: Evaluación del Nivel de satisfacción presentado por los pacientes operados por Lipoinyección con Grasa Autóloga.

\begin{tabular}{|c|c|c|}
\hline $\begin{array}{l}\text { Recibió Información después de } \\
\text { la intervención quirúrgica. }\end{array}$ & Frecuencia & Porcentaje \\
\hline No satisfecha(o) & 0 & 0 \\
\hline Si satisfecha(o) & 23 & 100 \\
\hline Total & 23 & 100 \\
\hline $\begin{array}{l}\text { Satisfecha con los cuidados del } \\
\text { cirujano }\end{array}$ & Frecuencia & Porcentaje \\
\hline No satisfecha(o) & 0 & 0 \\
\hline Si satisfecha(o) & 23 & 100 \\
\hline Total & 23 & 100 \\
\hline $\begin{array}{l}\text { Satisfecha con la forma de sus } \\
\text { labios }\end{array}$ & Frecuencia & Porcentaje \\
\hline No satisfecha(o) & 2 & 8,7 \\
\hline Si satisfecha(o) & 21 & 91,3 \\
\hline Total & 23 & 100 \\
\hline $\begin{array}{l}\text { Satisfecha con el volumen de sus } \\
\text { labios }\end{array}$ & Frecuencia & Porcentaje \\
\hline No satisfecha(o) & 2 & 8,7 \\
\hline Si satisfecha(o) & 21 & 91,3 \\
\hline Total & 23 & 100 \\
\hline $\begin{array}{c}\text { Satisfecha con la simetría de sus } \\
\text { labios }\end{array}$ & Frecuencia & Porcentaje \\
\hline No satisfecha(o) & 1 & 4,3 \\
\hline Si satisfecha(o) & 22 & 95,7 \\
\hline Total & 23 & 100 \\
\hline $\begin{array}{l}\text { Satisfecha con la consistencia de } \\
\text { sus labios }\end{array}$ & Frecuencia & Porcentaje \\
\hline No satisfecha(o) & 1 & 4,30 \\
\hline Si satisfecha(o) & 22 & 95,7 \\
\hline Total & 23 & 100 \\
\hline Grado de sensibilidad Labial & Frecuencia & Porcentaje \\
\hline No satisfecha(o) & 0 & 0 \\
\hline Si satisfecha(o) & 23 & 100 \\
\hline Total & 23 & 100 \\
\hline Nivel de satisfacción global & Frecuencia & Porcentaje \\
\hline Alto de satisfacción & 19 & 82,70 \\
\hline Medianamente satisfecha(o) & 4 & 17.39 \\
\hline $\begin{array}{r}\text { Bajo nivel de satisfacción o } \\
\text { insatisfacción. }\end{array}$ & 0 & 0 \\
\hline
\end{tabular}

Elaboración propia. 
La tabla No.6 muestra la encuesta aplicada a los tres meses para evaluar el grado de satisfacción del paciente con Cirugía Estética por Lipoinyección, los ítems evaluados respecto al nivel de información recibidos y al cuidado recibido durante el proceso hasta la actualidad en ambos hay un $100 \%$ de satisfacción.

En lo referente a la forma de los labios 2 de los pacientes que representan el $8.69 \%$ del total expresaron no estar satisfechos y $21(91.30 \%)$ expresaron estar satisfechos.

Respecto al volumen $2(8.69 \%)$ refirieron no estar satisfecho y 21(91.30) estar satisfecho.

En lo relativo a la simetría y consistencia hubo 1(4.34 \%) paciente por cada una de estas complicaciones que quedó no satisfecho.

A modo de resumen 6 pacientes mostraron algún indicador específico de los ya descritos de no satisfacción, lo que representa el $26.08 \%$ del total de la muestra y el $73.92 \%$ dijo estar satisfecho.

\section{DISCUSIÓN}

Considerando los resultados de esta investigación en lo que respecta a la edad y el sexo muestran que no es infrecuente que a medida que aumente la edad se soliciten este tipo de cirugía, Estos datos son coincidentes con los encontrados por la ISAPS(3) y ASAPS(11) en el 2018 que apuntan que a nivel mundial se realizan más Cirugías Plásticas Estéticas en mujeres que en hombres.

La mayor parte de las veces la solicitud de CP respecto a la edad y el sexo se asocia al envejecimiento, este dato es coincidente con varios estudios que así lo plantea, Scardovi, et al.(12) (2017) señalaban que los pacientes entre 40 y 60 años de edad acuden muchos más a las cirugías del tipo de rejuvenecimiento que otras edades entre ellas por labios finos, flácidos e hipotónicos, queilitis angulares y pérdida del soporte natural de tejidos por envejecimiento, ya que el proceso de envejecimientoen la piel trae aparejado pérdidas de vascularización, de reproducción celular (fibroblastos), fibras colágenas y elásticas y fundamentalmente de Ácido Hialurónico, lo cual conlleva a la deshidratación y pérdida de volumen $(8,12)$.

Planas Pavón, et al.(13) realizaron un estudio donde apuntan que desde la antigüedad hasta nuestros días las personas buscan diferentes métodos incluidos la cirugía, para rejuvenecer su rostro, de ahí que la edad se asocie a la pérdida de la belleza, la cual se fundamenta en la armonía de rasgos y facciones, así como en la existencia de características asociadas a la juventud. Razón por la que el mayor número de pacientes en este estudio se encuentre entre 51 y 61 años de edad.

En Cuba que es un país con una población altamente envejecida pensar en ello se convierte en una necesidad y en un problema de salud que no solo ataña a las enfermedades crónicas o de otro tipo, sino también al bienestar físico y psicológico que se asocian a los cánones de belleza de ahí que Castiñeira López y Machado Martínez(14) refieran que por el aumento en solicitudes de pacientes ya adultos además de los tratamientos ya existentes, en la ortodoncia se incluya en los tipos de tratamientos para el envejecimiento y planteaba:

"El envejecimiento es un proceso que involucra no solo los tejidos blandos faciales sino además a las estructuras óseas, el mismo puede estar provocado por factores intrínsecos y extrínsecos... el tratamiento de Ortodoncia, puede prolongar la apariencia joven de un paciente o acelerar la aparición del envejecimiento. Constituye esta una temática que necesita un abordaje pedagógico curricular, en la formación de los especialistas en Ortodoncia" (14).

Cuba ha tenido un comportamiento similar al resto del mundo y al representado en la muestra de esta investigación, tanto en aumento de número de casos como en el predomino de mujeres por encima de los hombres, según datos divulgados en el 2do. Congreso de la Sociedad Cubana de Cirugía Plástica y Caumatología —realizado del 5 al 7 de septiembre 2018 en la Isla-, desde 2001 hasta 2016 se realizaron 309469 cirugías plásticas en Cuba, de las cuales 200.508 fueron cirugías estéticas, y las demás reconstructivas siendo en su mayoría mujeres las que optan por estos procederes(15).

Para los pacientes de esta muestra la edad promedio fue de 42.17 años, esta edad se corresponde con el grupo etario de 40 a 50 años, lo que permite anotar que la mayoría de los pacientes están alrededor de esta media y representan el $39.13 \%$ del total. Estos datos son coincidentes con un estudio realizado en el 2016 por Cosmetic Surgery National Data Bank Statistics, de la The American Society for Aesthetic Plastic Surgery (Sociedad Americana de Cirugía Plástica Estética)(16). y es coherente con todo lo planteado anteriormente pues a partir de los 40 años se puede apreciar por medios físicos e histológicos los signos de envejecimiento(8).

En los grupos de edades comprendidos entre los 18 a 39 años, para ambos sexos suman 11 pacientes lo que representan el 47 . $82 \%$ del total de la muestra. Es importante señalar que, para estas edades, la tecnología, el uso de los medios audiovisuales dentro de las redes cibernéticas, han adquirido un valor de dictaminación sobre patrones estéticos, culturales que son hoy decisivos a la hora de optar por la experiencia de una Cirugía Plástica Estética.

Referido a ello Lievano Franco(17) expone: “... una noción del cuerpo definida principalmente, a partir de la anatomía y por ende de un cuerpo fisico en el que está ausente el sujeto que lo porta". Es decir, el cuerpo se convierte en un proyecto en sí mismo, con fines y metas específicas, que pasan desde la sensualidad hasta la satisfacción del bienestar físico que pasa por la autoimagen y el cuidado obsesivo del cuerpo. 
Resulta importante resaltar que sobre todo para las edades comprendías antes de los 40 años se configura toda una dinámica social, de marketing, la internet, la página de oferta de cambios de físico, que permiten reconstruir el cuerpo disolviendo las limitaciones físicas, psicológicas y sociales hasta acceder a lo que se suponía inaccesible porque genéticamente le venía dado. Y nos referimos a estas edades pues a partir de los 40 esa misma dinámica social sobre la imagen adquiere otro tratamiento, y se proporciona a partir de los signos de envejecimiento asociados al recuerdo de la juventud.

Los labios adquieren una posición privilegiada en el rostro y son afectado por la edad, cambian la imagen y se asocian hasta razones sexuales, de ahí que los defectos labiales sean por la edad o de otros tipos, mediatizan la belleza o juventud, estos argumentos quedan bien descritos en Baldín, et al.(18), Scardovi, et al.(12) y Castiñeira López(15), cuando de manera similar aluden a que el labio es un órgano de complejas relaciones anatómicas importantes, funciones fisiológicas y grandes connotaciones sociales, cosméticas y sexuales.

Los defectos labiales se pueden asociar a razones de varios tipo entre ellas(accidentes, consecuencias post quirúrgicas, queloides entre otras) y del tipo genéticas o constitucionales que son las que se observan en esta investigación (labios finos) y las propias del proceso involutivo asociado a la edad (pérdida del volumen), pero ambas razones no justifican por sí mismas la solicitud de una Cirugía Plástica Estética, en opinión de este autor, más allá del sexo biológico y avalado por la bibliografía revisada, en los dos casos existen razones estéticas sustentadas por cuestiones culturales asociadas a efectos psicológicos, Baldin, et al.(18) platean en una revisión bibliográfica que:

"La insatisfacción con la autoimagen corporal es el principal factor para la búsqueda de procedimientos estéticos, y la Cirugía Plástica es un componente esencial para la restauración del aspecto psicológico del paciente, mejorar la integración con su entorno biopsicosocial y proporcionar bienestar y autoconfianza ... existen estudios que demuestran que hasta un $48 \%$ de pacientes que solicitan atención por un cirujano plástico padecen algún tipo de trastorno psicológico, sin importar el grado de afectación, y buscan en la CP una oportunidad para lograr su satisfacción"

Ya realizada la cirugía, esta viene acompañada de reacciones propias del proceder quirúrgico, sean estas inmediatas o tardías no son infrecuentes, los resultados obtenidos aquí son coincidentes con los encontrados por Salgado Roa(19) en su tesis con pacientes operados bajo esta misma técnica y lugar anatómico, el cual encontró que el edema en los labios y el hematoma aparecían en un $8.3 \%$ en su muestra. El Centro de Estética adjunto a la ASPAS en el 2016(20), afirmaba que la reacción inflamatoria aguda es una de las complicaciones más frecuentes encontradas.

Otro estudio realizado por Scardovi(12) et al describían en su investigación, que de los 27 pacientes en el 67,5\%, no hubo complicaciones de ningún tipo y que en 13 pacientes el 32,5\% de los casos tratados hubo complicaciones inmediatas y que remitieron entre 1 y 15 días en algunos de los 13 casos hubo: Hematomas (11), Induración (3), Inflamación con/sin enrojecimiento (2), Edema(2), Nódulo( 1) -deposición fuera de plano del material- y Dolor (1)

No obstante, se señalan otras complicaciones inmediatas que no se dieron en los pacientes de esta investigación, el autor de este trabajo considera que quizás se deba al tamaño de la muestra, al ser esta muy pequeña por lo que no es coincidente con lo referido en otras publicaciones como las de Scardovi12 y Vélez- Benítez21 que aluden otras como eritemas, efectos alérgicos, hipertermia, entre otras.

Los resultados encontrados sobre las complicaciones tardías son coincidentes con los encontrados por Scardovi(12), VélezBenitez(21), que referirán los trastornos de la sensibilidad, el endurecimiento y las nodulaciones en este tipo de cirugía, no son infrecuentes.

Dos artículos de revisión resultaron muy importantes para este tópico y fueron los de Mundada(22) et al, que planteaba: "las complicaciones tardías son relacionadas como una respuesta propia al proceder, estas complicaciones incluyen granulomas por cuerpo extraño, manifestaciones tardías que incluyen formación de accesos, migración del relleno, nódulos, tejido que se puede necrosar e inclusive ulcerar y descoloraciones persistentes"

Y este segundo de Urdiales(23) et al, donde se reunieron un grupo multidisciplinario de expertos a realizar esta revisión en noviembre del 2016 en aras de discutir y conveniar cuáles eran las principales complicaciones del uso de rellenos incluidos la grasa autóloga como principal en la Cirugía Estética, señalando que dentro de las tardías estaban: el Angioedema, que se puede observar desde las primeras horas, pero puede en algunos casos durar por más de 6 semanas. Otro tipo de edema (tardío) que se observa como una reacción tardía de hipersensibilidad caracterizada por induración, eritema y edema que ocurre usualmente desde el primer día después de la inyección y que puede persistir por meses.

Otro tipo de edema es el Malar es una complicación que ha sido reportada como frecuente y se asocia a la inyección infraorbital, otro síntoma de complicación tardía es la descoloración de la piel, la infección, los abscesos y los nódulos y muy raro es la inminente necrosis del tejido injertado, muchas de las complicaciones, no se dieron en los pacientes operados, una primera causa es que no siempre se dan todos, ello dependerá de la propia manipulación del tejido y de una respuesta anatómica "común" a esta, como la respuesta inflamatoria aguda entre otras(23).

Resulta llamativo que las respuestas tardías alcanzan gran número de síntomas y porcientos entre la población sometida a 
este tipo de cirugías según varios autores como los enunciados anteriormente. Como ya se hubo de expresar, la falta de representatividad de la muestra es en nuestra opinión el factor fundamental al pequeño número de complicaciones inmediatas y tardías encontradas en este trabajo

El estado de satisfacción de los pacientes está descrito en la literatura sobre todo referidos a parámetros como: la forma, consistencia y volumen pues muchas veces forman parte de la subjetividad del mismo y no necesariamente de la técnica quirúrgica empleada pues suelen esperar "algo mágico" y perfecto, lo que no siempre depende de la experiencia del cirujano, sino de las expectativas de los pacientes(24). La CP es un área en que los resultados reportados por los pacientes son particularmente importantes, dado que la evaluación de fotos antes y después por los cirujanos no se correlaciona con satisfacción/experiencias de los pacientes(25).

Gómez Zayas(9) coincide con lo antes referido cuando plantea en su investigación: "Haber obtenido un porcentaje alto en esta investigación, no solamente está en relación con los detalles propios de la técnica empleada sino además con la explicación sobre las ventajas y desventajas del proceder a los pacientes para no crear falsas expectativas en ellos".

No obstante, más allá de las expectativas del paciente es cierto que existen factores relacionados al proceder quirúrgico como el manejo de la técnica, lo cual va a depender de la práctica sistemática, por ejemplo, el manejo de la cantidad de volumen de tejido graso a inyectar, la presión en la manipulación o extracción de la grasa en términos de presión ejercida y otros factores los cuales mediatizan el resultado final y por supuesto los niveles de satisfacción, e igual, estos parámetros no se evaluaron en este trabajo, no obstante se siguieron y describieron los pasos en el proceder( material y métodos) con que se actuó en esta técnica para garantizar un buen resultado.

Existen otros factores que pudieran estar influyendo en la percepción de la satisfacción según Blanco Moredo(26) et al: los labios son zonas de gran movilidad, lo que no ayuda en las primeras 48 horas a la revascularización del tejido graso injertado y puede afectar la sensación de consistencia y el volumen.

A modo de conclusión el autor de esta investigación considera que:

- Dentro de los objetivos de este estudio no estuvo evaluar las posibles causas del porqué estos niveles de satisfacción relacionados con el resultado quirúrgico final, pero se puede inferir que algunos de estos pudieron estar mediatizado por las expectativas del paciente sobre todo en relación a la forma, volumen y la consistencia, tres parámetros que pasan de manera importante por la subjetividad de los mismos, sin descartar (pero dándole un peso mucho menor) el desempeño en el proceder quirúrgico.

- A saber, que las complicaciones no solo dependen de la práctica sistemática, de buenas prácticas, desempeño del cirujano o factores como la anatomía referidas anteriormente. Están también los cuidados post operatorios por parte del paciente, los cuales son esenciales, al ser esta una cirugía rápida, limpia, no compleja, pudiera existir una tendencia por parte de estos a minimizar los riesgos post operatorios relacionados con los cuidados necesarios, los mismos que se asocian al éxito.

- La discusión realizada esta limitada por la bibliografía escasa acerca del tema específico de Lipoinyección Labial Facial, el autor tuvo que utilizar bibliografía similar para comparar sus resultados con técnicas semejantes y/o la anatomía facial general. No obstante, estos resultados y la discusión, dan una visión cercana a la evidencia médica de la práctica cotidiana, lo cual resultará útil, más allá de que la muestra haya sido pequeña

Los resultados y la discusión de este trabajo logran denotar la importancia de la técnica empleada, la necesidad de seguir evaluando la satisfacción como medidor de calidad desde la actuación médica del cirujano y desde cómo evalúa o percibe el resultado final el paciente que es lo más importante.

\section{CONCLUSIONES}

La Lipoinyección labial con Grasa Autóloga se caracterizó por ser un método capaz de satisfacer el resultado estético en los pacientes con labio hipotrófico obteniéndose un alto nivel de satisfacción.

Las variables edad y sexo evidencian que las mujeres se realizaron más Cirugía Estética Labial que los hombres y que a medida que aumentan los signos de envejecimiento por la edad este proceder se convierte en un tratamiento de elección para ambos sexos.

Las variables clínicas labios finos y volumen evidencian que el aumento de la edad es una condición importante que se asocia a la demanda de esta técnica quirúrgica y que el ser hombre o mujer no establece una diferencia para la solitud de este tipo de técnica quirúrgica.

Se presentaron complicaciones inmediatas y tardías que no difieren de las encontradas en otras investigaciones, las cuales no afectaron el resultado final en términos de satisfacción global con el proceder quirúrgico.

Los niveles de satisfacción expresados por los pacientes fueron altos, obteniéndose estéticamente un cambio favorable.

Conflicto de Interés: Los investigadores declaran no tener ningún conflicto de interés en la realización del estudio.

Agradecimientos: A mi tutora la Dra. Dayamí Zaldívar, educadora de extraordinarios valores humanos, a mi asesora la Dra. Clara Elena Peñalver Rodríguez, a mi profesora y amiga, por su excelente sentido humanista y al Consejo de Dirección 
del Clínico Quirúrgico “General Calixto García” por confiar en mí y su apoyo para el desarrollo de esta investigación.

\section{REFERENCIAS}

1. Sánchez Navarro, Z. J. y Nagles López, L. V. (2018). Desde el punto de vista jurisprudencial ¿la cirugía estética es una actividad medios o de resultado? (Tesis de pregrado). Recuperado de: http://repository.ucc.edu.co/handle/ucc/7396

2. Sociedad Colombiana de Cirugía Plástica, Estética y Reconstructiva [en línea].Bogotá: ISAPS. [Actualizada Octubre 2018; citado 15 febrero 2020]. Historia de la cirugía plástica. Recuperado de: https://www.cirugiaplastica.org.co/quienes- somos/historiade-lacirugia-plastica.html

3. Encuesta internacional anual sobre procedimientos estéticos \cosméticos 2018. Hanover, Nuevo Hampshire.3 de Dic de 2019

4. Fernández Díaz J. Operaciones estéticas más demandadas en Cuba. Cuba en Miami. 4 de octubre 2018; Temas calientes: 1

5. Suárez-Oyhamburú Darío L, Escobar-Ugarte Raúl A. Uso de matriz dérmica autóloga en reconstrucción mamaria, un recurso accessible. Cir. plást. iberolatinoam. [Internet]. 2018 Jun [citado 2021 Mar 22] ; 44( 2 ): 169-176. Disponible

en: http://scielo.isciii.es/scielo.php?script=sci_arttext\&pid=S03 76-78922018000200009\&lng=es. Epub 08-Feb-2021. http://dx.doi.org/10.4321/s0376-78922018000200009.

6. Gal S, Xue Y, Pu LLQ. What Do We Know Now About Autologous Fat Grafting? Ann Plast Surg. 2019 Oct;83(4S Suppl 1):S17-S20. doi: 10.1097/SAP.0000000000002097. PMID: 31513062.

7. Ruiz-Matta JM, Peniche-Castellanos A, Fierro-Arias L, et al. Aumento de mentón mediante implante de grasa autóloga abdominal. Dermatol Rev Mex. 2017;61(3):190-196.

8. Tenjo Aguirre EM, Silva Briones GA. Efectividad de la aplicación de Células Madre y Factor de Crecimiento Plaquetario en el tratamiento de mujeres de 40 a 60 años que presenten Involución Cutánea facial, atendidas en la unidad de medicina y cirugía estética Renove de la ciudad de Guayaquil 2016-2017.[Tesis de licenciatura].Ecuador: Universidad Católica de Santiago de Guayaquil, Facultad de Ciencias Médicas; 2017

9. Gómez Zayas O. Rejuvenecimiento mediofacial con microinjertos de grasa autóloga enriquecida con Plasma Rico en Plaquetas. Hospital General "Calixto García". 2012 - 2014. [Tesis de especialidad médica]. Cuba. Universidad de Ciencias Médicas de la Habana. 2014

10. Manzini Jorge Luis. DECLARACIÓN DE HELSINKI: PRINCIPIOS ÉTICOS PARA LA INVESTIGACIÓN MÉDICA SOBRE SUJETOS HUMANOS. Acta bioeth. [Internet]. 2000 Dic [citado 2021 Mar 22] ; 6( 2 ): 321$334 . \quad$ Disponible en: https://scielo.conicyt.cl/scielo.php?script=sci_arttext\&pid= S1726-569X2000000200010\&lng=es. http://dx.doi.org/10.4067/S1726-569X2000000200010.

11. Torres Pérez, S., \& Vargas Rojas, M. (2017). Estilo de vida saludable en mujeres adolescentes consumidoras de cirugías plásticas. (Trabajo de grado). Villavicencio: Universidad Cooperativa de Colombia.

12. Scardovi S, Goglian A, Gendra P, Gendra C. Estudio clínico de eficacia, duración y efectos adversos del implante de
Ácido Hialurónico en el área Buco-Maxilo-Facial. ode [Internet]. 8mar.2019 [citado 22mar.2021];19(30):77-0. Available from: https://www.odon.edu.uy/ojs/index.php/ode/article/view/16 $\underline{6}$

13. Planas Pavón M, González Piedra M, Zamora Santiesteban Y, Fuentes Céspedes O. Aplicación del plasma rico en plaquetas en la especialidad de cirugía plástica y caumatología. Invest Medicoquir. 2019;11(Suppl: 1):.

14. Castiñeira LD, Machado MM. La inclusión del tema envejecimiento facial en el proceso de enseñanzaaprendizaje de la Ortodoncia. Gaceta Médica Espirituana. 2017;19(1):.

15. Domínguez Cruz AM. Cirugía plástica en Cuba: ¿Juventud y belleza a toda costa? Juventud Rebelde.29 Sep 2018; Salud

16. Cosmetic Surgery National Data Bank Statistics. ASAPS. 2016; Recuperado de http://www.surgery.org/sites/default/files/ASAPSStats2016.pdf

17. Lievano Franco MP. El uso de la Cirugía Estética: un acercamiento a la reconstrucción del cuerpo y la subjetividad femeninos [tesis doctoral]. Bellateras: Universidad Autónoma de Barcelona, Facultad de Psicología; 2012

18. Baldin AV, Telich-Tarriba JE, Díaz-Lozano DJ, et al. Depresión y cirugía plástica. Cir Plast. 2018;28(1):3-7. doi:10.35366/CP181A.

19. Salgado Roa E. Aumento de labios con la técnica de Lipoinyección de grasa autóloga. [Tesis de especialidad médica] Cuba. Universidad de Ciencias Médica de la Habana. 2003

20. American Society of Plastic Surgeons. Specific Risks of Fat Graft or Fat Transfer Procedures. ASPS. [en línea].2016. [citado 4 Ene 2020].URL disponible en: http://www.aestheticcenterhawaii .kaiserpermanente.org

21. Vélez;Benítez E, Cuenca;Pardo J, Ramos;Gallardo G, et al. Seguridad en la aplicación de rellenos faciales. Medicina basada en evidencia. Cir Plast. 2019;29(1):67-87. doi:10.35366/91493.

22. Mundada P, Kohler R, Boudabbous S, ToutousTrellu L, Platón A, Becker M.Injectable facial fillers: imaging features, complications, and diagnostic pitfalls at MRI and PET CT. Insights Imaging [en línea]. 2017 [citado 15 Ene 2020]; 8:557-572.doi: 10.1007/s13244-017-0575-0

23. Urdiales Gálvez F, Escoda Delgado N, Figueiredo V, Lajo Plaza JV, Mira M, Moreno A. Treatment of Soft Tissue Filler Complications: Expert Consensus Recommendations. Aesthetic Plast Surg.2018 Apr;(42)2:498-510

24. Clustersalud.com, Gestión Hospitalaria [en línea]. España: EurekAlert; 2016. [citado 18 Ene 2020]. Disponible en: https://clustersalud.americaeconomia.com/tags/cirujanosplasticos

25. Claudia R Albornoz G, Andrea L Pusic, Stefan Danilla E., evaluación de resultados quirúrgicos desde la perspectiva del paciente, Revista Médica Clínica Las Condes, Volume 27, Issue 1, 2016, Pages 107-112, ISSN 0716-8640, https://doi.org/10.1016/j.rmclc.2016.01.013

26. Blanco-Moredo E, Dunán-Mesa LY, Pérez-Ferrer MS. Lipotransferencia: una alternativa para el tratamiento de la deformidad facial adquirida. RIC. 2020;99(1):63-70. 